Article

\title{
Simvastatin Release from Poly(lactide-co-glycolide) Membrane Scaffolds
}

\author{
Hassan Rashidi ${ }^{1}$, Marianne J. Ellis ${ }^{1}$, Sarah H. Cartmell ${ }^{2}$ and Julian B. Chaudhuri ${ }^{1, *}$ \\ 1 Centre for Regenerative Medicine, Department of Chemical Engineering, University of Bath, \\ Claverton Down, Bath, BA2 7AY, UK; E-Mails: mgxhr@nottingham.ac.uk (H.R.); \\ M.J.Ellis@bath.ac.uk (M.J.E.) \\ 2 School of Materials, Materials Science Centre, University of Manchester, Grosvenor Street, \\ Manchester M13 9PL, UK; E-Mail: sarah.cartmell@manchester.ac.uk (S.H.C.) \\ * Author to whom correspondence should be addressed; E-Mail: J.B.Chaudhuri@bath.ac.uk; \\ Tel.: +44-1225-386349; Fax: +44-1225-385713.
}

Received: 15 November 2010; in revised form: 30 November 2010 / Accepted: 8 December 2010 / Published: 9 December 2010

\begin{abstract}
Statins, a group of potent inhibitors of 3-hydroxy-3-methylglutaryl Coenzyme A reductase in cholesterol biosynthesis pathway, have been widely used as a cholesterol lowering drug. The plieotrophic effect of statins on bone metabolism in long-term usage has been begun to be studied during recent years and several in vitro and in vivo studies have demonstrated the ability of statins to promote expression of bone morphogenetic protein-2 (BMP-2), inhibition of osteoclast differentiation and reduction of osteoporotic fractures risk. The high liver specificity and low oral bioavailability of statins, leading to poor peripheral distribution, are the main obstacles to benefit anabolic effects of hydrophobic statins on bone formation. Therefore, developing new administration roots for direct delivery to achieve optimum concentration in the bone microenvironment is of interest. Here we present and compare two approaches of combining statins with bone tissue engineering scaffolds. Simvastatin was combined with a poly(lactide-co-glycolide) (PLGA) membrane scaffold for diffusion-controlled release by dissolving simvastatin (dis-sim) in the membrane casting dope, and for degradation-controlled release by covalently bonding saponifiedsimvastatin (sap-sim) to the PLGA in the spinning dope. Rheological and concentration-dependent membrane morphology changes were observed with saponifiedsimvastatin, suggesting ester bond cleavage and covalent bonding of the statin to the PLGA, but not with dissolved simvastatin. Dissolved simvastatin membranes
\end{abstract}


showed a logarithmic decay release profile while the saponifiedsimvastatin membranes showed constant release. It can be concluded that the covalent bonding of simvastatinto PLGA scaffolds is showing potential for use as a controlled releasescaffold for bone tissue engineering.

Keywords: PLGA; membrane; scaffold; statin; controlled release; bone tissue engineering

\section{Introduction}

More than one in three women and one in ten men suffer from osteoporosis over a lifetime, with a fracture risk of up to $40 \%$ in women and 13-25\% in men [1]. Osteoporosis is the most common debilitating skeletal disorder, characterized by declining quality and quantity of cancellous and cortical bone [1]. The compounds that are currently available for the treatment of osteoporosis are mainly limited to anti-resorptive drugs such as calcitonin, estrogen, selective estrogen receptor modulators, biphosphonates, calcium supplementation and ipriflavone. Anti-resorptive drugs inhibit osteoclast activity and decrease bone turn over but lack anabolic effect with a very weak to moderate effect on elevation of bone mineral density (BMD) which decreases to less than $50 \%$ in advance stages of osteoporosis. Parathyroid hormone (PTH), sodium fluoride and strontium ranelate are few candidates currently under investigation with anabolic effects on bone metabolism, however their therapeutic application faces number of issues of concern [1]. In addition to some gastrointestinal side effects, the result of using fluoride in clinical trials were not encouraging despite its initial promise [2,3]. Apart from cost, root of administration, dosage regimen and its long-term effect on bone are questions that need to be answered before using PTH as bone anabolic agent in the clinic [1]. Strontium ranelate is another compound currently under investigation with bone anabolic effects and has shown a dual action on bone metabolism by reduction of bone resorption and stimulation of bone formation both in vitro [2] and in vivo [3].

A group of drugs called statins were introduced as cholesterol-reducing drugs but have been found to increase bone mass [4-6]. Statins are irreversible and competitive inhibitors of 3-hydroxy-3-methylglutaryl Coenzyme A (HMG-CoA) reductase that initially acts by displacing the natural substrate (HMG-CoA) in the cholesterol biosynthetic pathway, reducing biosynthesis of mevalonate and consequently cholesterol in a dose-dependent manner [1,7]. Some beneficial effects of statins are related to their hypocholesterolemic property and inhibition of HMG-CoAreductase such as prevention of arthrosclerosis. However, other beneficial effects related to administration of statins have been reported that are not in direct relation with cholesterol reduction, a phenomenon known as a pleiotropic effect. Amongst the various pleiotropic effects of statins, their anabolic effect on bone metabolism, reported for the first time in 1994, has recently been receiving attention. Although the exact molecular mechanism of increase in bone formation remains elusive [1], the collected in vitro and in vivo evidence supports the notion that stimulation of vascular endothelial growth factor (VEGF) [8] and bone morphogenic protein-2 (BMP-2) due to possible inactivation of functional prenylated small GTPase produced in the mevalonate pathway is responsible for the anabolic effects of statins on bone metabolism [6,9-11]. In vitro induction of osteogenesis on a non-transformed 
osteoblast (MC3T3-E1) with simvastatin revealed that induction of BMP-2 expression and enhancing alkaline phosphatase activity and mineralization take place in a dose- and time-dependent manner [11]. In addition, anti-resorptive properties and inhibition of osteoclast formation have been attributed to the statins in a number of studies [4,12]. In contrast to in vitro studies which all affirm the anabolic and anti-resorptive effects of statin in bone metabolism, the results of in vivo studies have been controversial. While a number of studies using subcutaneous and oral administration of mainly lipophilicstatins have claimed a significant increase in new bone formation in normal and ovariectomizedrats [13,14], others have found more controversial results, indicating a lack of anabolic effects on bone formation [15,16] and an increase in bone turnover and decrease in bone formation [17]. It has been suggested that poor absorption of statins (less than $5 \%$ of an oral dose), high hepatic selectivity and first-pass effect cause a low systemic and peripheral concentration of statins following by oral administration [1,14]. Consequently, osteoblasts and osteoclasts exposed to a very low concentration of statins which might not be sufficient for their alteration, indicate that oral administration is not an ideal delivery method to benefit possible anti-osteoporotic property of statins [1] while significant increase in new bone formation was seen following topical [18,19], subcutaneous [4] and direct injection into the bone marrow cavity [20] compared to oral administration. New techniques for the administration of statins, for promotion of osteogenesis, have utilisedstatin-embedded polymers, manifesting significant positive effects on bone formation [21-24]. Although the effect of statins, especially the lipophilic derivatives on elevation of BMP-2, increase of ALP activity and promotion of osteogenesis have been proven in numerous studies, little has been done to capitalize on their effect by developing a suitable scaffold (a biomaterial that can be used to implant into a patient defect) for promotion of osteogenesisin vitro. In addition, scaffolds can be used as suitable delivery devices to overcome low concentration of statins in peripheral blood circulation necessary for anabolic effect on bone metabolism by delivering the drug locally. Scaffolds are highly porous structures which provide a three-dimensional support for cell attachment, proliferation and differentiation [25] to ultimately aid regeneration of an injured site. Polymer structures have been widely used to develop new controlled release drug delivery devices and devising such a drug delivery device to deliver statins to the site of injury at the desirable local drug concentration is necessary to fully utilize the anabolic effects of statins. The in vitro effect of statins on formation of bone have been known for more than half decade, however it is only recently that two polymer-based biomaterials have been developed by grafting simvastatin into the PLGA using oxalyl chloride chemical reaction [20] and incorporating fluvastatin into poly(ethanol glycol) based hydrogel [26]. In order to develop new controlled release systems to be used in bone tissue engineering, grafting statins into polymer via covalent bonds is ideal to achieve a degradation-dependant release instead of a diffusion-dependant release, characteristic of drug-blended polymers.

Simvastatin is currently the most commonly prescribed statin in the UK and is available for purchase in a pharmacy without prescription. It has been shown that simvastatin significantly up-regulates osteoblast differentiation and extracellular matrix production [11].

We are investigating the approach of combining simvastatin covalently with poly(lactide-co-glycolide) (PLGA) to develop a controlled release system for simvastatin, in a biocompatible and degradable polymer that is suitable for implantation into bone tissue. PLGA has a long history of safe and effective clinical use.Using PLGA to support and guide a wound healing response in a bone defect is a 
therapeutic option. Combining PLGA with a bone-stimulating molecule such as simvastatin could greatly enhance the local physiological repair mechanism. This paper outlines a method to covalently attach simvastatin to PLGA using saponificationand describes our results on the subsequent controlled release patterns.

\section{Experimental Section}

\subsection{Materials}

Poly(D,L-lactide-co-glycolide), having a lactide:glycolide molar ratio of 75:25, (PLGA) was purchased from BoehringerIngelheim (RG756S, Ingelheim, Germany), 1-methyl-2-pyrrolidinone (NMP) and ethanol absolute 99.8+\% was purchased from Acros Organics (Geel, Belgium); NMP was reagent grade and PLGA was GMP grade and ethanol was GLC grade. ACN and acetic acid were purchased from Sigma-Aldrich, UK. Simvastatin was purchased from Johnson and Johnson.

Simvastatin was used in its unmodified form, with the lactone ring intact, and dissolved in the membrane casting dope for diffusion-dependent release; for degradation-dependent release, simvastatin was saponified (Figure 1) then added to the membrane casting dope for covalent bonding to the PLGA. The scaffold preparation is described below for both the dissolved simvastatin (dis-sim) and the saponifiedsimvastatin (sap-sim).

Figure 1. Chemical structure of simvastatin with the closedlactone ring prior to- and open lactone ring after the process of saponification.
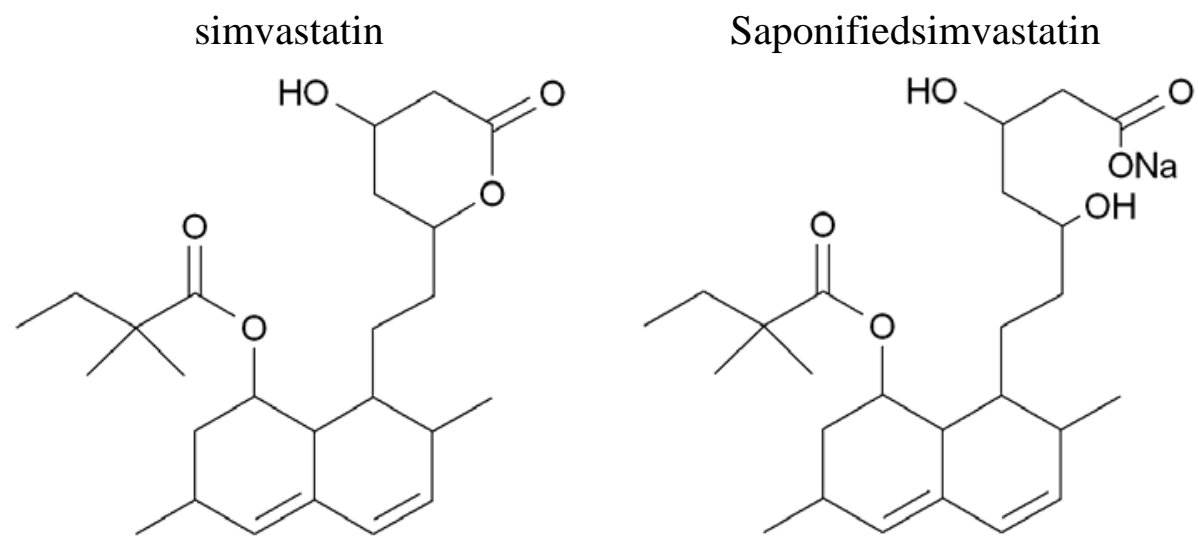

\subsection{Extraction and Saponification of Simvastatin}

10 mg simvastatin tablets were saponified using a methoddeveloped by Whang et al. [19]; a mixture of EtOH extracted solution of simvastatin with $0.1 \mathrm{~N}$ sodium hydroxide $(\mathrm{NaOH})$ was heated at $50{ }^{\circ} \mathrm{C}$ for two hours.

\subsection{Membrane Scaffold Preparation}

The PLGA membrane casting dope was prepared from PLGA and NMP in a $20 \%(\mathrm{w} / \mathrm{w})$ polymer solution. For diffusion-controlled release, dis-sim was dissolved in the casting dope at concentration of $1 \mathrm{mg}$ simvastatin per g PLGA (mg/g). For degradation-controlled release, sap-sim at two different 
concentrations of $0.5 \mathrm{mg} / \mathrm{g}$ and $1 \mathrm{mg} / \mathrm{g}$ were dissolved in the casting dope. Flat-sheet membrane scaffolds were prepared by solvent exchange with distilled water as described elsewhere [26,27].

\subsection{Simvastatin Quantification by HPLC}

HPLC analysis was carried on a Shimadzu LC-10A series system (Kyoto, Japan) equipped with a Phenomenex AQUA C18 reverse phase column $(150 \mathrm{~mm} \times 4.6 \mathrm{~mm}, 5 \mathrm{~mm}$; Torrance, USA). A mixture of filtered and degassed $\mathrm{ACN}: \mathrm{H}_{2} \mathrm{O}+0.1 \%$ Acetic acid $(60: 40 \mathrm{v} / \mathrm{v})$ was used as mobile phase and flow rate of $1.0 \mathrm{~mL} / \mathrm{min}$ at max UV absorption of $248 \mathrm{~nm}$. In order to evaluate the kinetics of drug-release, samples were taken from the air-dried membranes and soaked in absolute $\mathrm{EtOH}$ to facilitate degradation of membrane.

\subsection{Viscosity of the PLGA Membrane Casting Dopes}

The viscosity of the PLGA membrane casting dopes was measured using a plate and cone rheometer (BohlinRheometer CS, BohlinReologi, Sjöbo, Sweden) at a constant temperature of $20{ }^{\circ} \mathrm{C}$.

\subsection{Morphology of PLGA Membrane Scaffolds}

The morphology of the simvastatin-free, dis-sim and sap-sim PLGA flat sheet membranes was studied using scanning electron microscopy (SEM) after 3 days in deionised water to remove residual solvent. Samples were dried before preparation for SEM. A thin layer of gold coating was deposited on the sample before observation with the SEM microscope (JSM6310, JEOL, Herts, UK) after sputtering with gold (5150B sputter coater, BOC Edwards, West Sussex, UK).

\section{Results and Discussion}

The overall objective of this study was to investigate techniques to incorporate simvastatin into PLGA membrane scaffolds and the resulting effects on the membrane properties and the release mechanism, to develop new membrane scaffolds to be used for bone tissue engineering purposes.

In a new approach, simvastatin was incorporated into PLGA flat sheet membrane scaffolds by adding either the unmodified drug (dis-sim), or saponifiedsimvastatin (sap-sim) to the PLGA membrane casting dope. Molecular weight and polymer chain structure are recognized as important factors governing polymer properties [18] and therefore characteristics such as the solution properties of the membrane casting dope, thermodynamic interactions with the solvent and non-solvent, and physical behaviour during casting which in turn determine the final membrane structure.

\subsection{Viscosity of the Casting Dopes Was Reduced on Addition of Sap-Sim}

To evaluate the effect of simvastatin on the rheology of the PLGA membrane casting dopes, viscosity measurements were taken before and after introduction of either dis-sim or sap-sim, the values of which are shown in Table 1 . A significant reduction in the viscosity of the membrane casting dope was seen when sap-sim dopes were compared to the drug-free dopes. Furthermore, a proportional relationship between viscosity of sap-sim dopes to the concentration of simvastatin in the dopes was observed. Drug-free dopes had a viscosity of $1.2 \mathrm{~Pa}$ s and an increase in sap-sim from $0.5 \mathrm{mg} / \mathrm{g}$ to $1 \mathrm{mg} / \mathrm{g}$ 
caused a reduction in viscosity from $0.2 \mathrm{~Pa} \mathrm{~s}$ to $0.05 \mathrm{~Pa} \mathrm{~s}$. The addition of dis-sim to the membrane casting dope did not have an effect on the viscosity which remained at 1.2 Pa s.

Table 1. Viscosities of membrane casting dopes.

\begin{tabular}{ccccc}
\hline Dope Composition & PLGA & $\begin{array}{c}\text { PLGA + Dis-Sim } \\
(1.0 \mathrm{mg} \mathrm{sim} / \mathrm{g} \\
\text { PLGA })\end{array}$ & $\begin{array}{c}\text { PLGA + sap-sim } \\
(0.5 \mathrm{mg} \mathrm{sim} / \mathrm{g} \\
\text { PLGA })\end{array}$ & $\begin{array}{c}\text { PLGA + sap-sim } \\
(1.0 \mathrm{mg} \mathrm{sim} / \mathrm{g} \\
\text { PLGA })\end{array}$ \\
\hline Viscosity (Pa s) & 1.2 & 1.2 & 0.21 & 0.05 \\
\hline
\end{tabular}

\subsection{Covalent Bonding of Sap-Sim to PLGA Appears to Increase Porosity of Membranes}

Scanning electron microscopy analysis revealed no difference in the PLGA membrane surface morphology when they were cast using drug-free casting dopes [Figure 2(A)] or dis-sim casting dopes [(Figure 2(B)]. With increasingaddition of sap-sim, an increase in the number and size of surface pores were observed [Figure 2(C,D)]. It was also observed that the increase in sap-sim gradually changed the PLGA membrane from flexible to brittle.

Figure 2. Surface morphology of PLGA membranes with different simvastatin preparations. (A) PLGA only, (B) PLGA + dis-sim (1.0 mg sim/g PLGA), (C) PLGA + sap-sim (0.5 mg Sim/g PLGA), (D) PLGA + sap-sim (1.0 mg Sim/g PLGA) 3 days after detoxification in water.
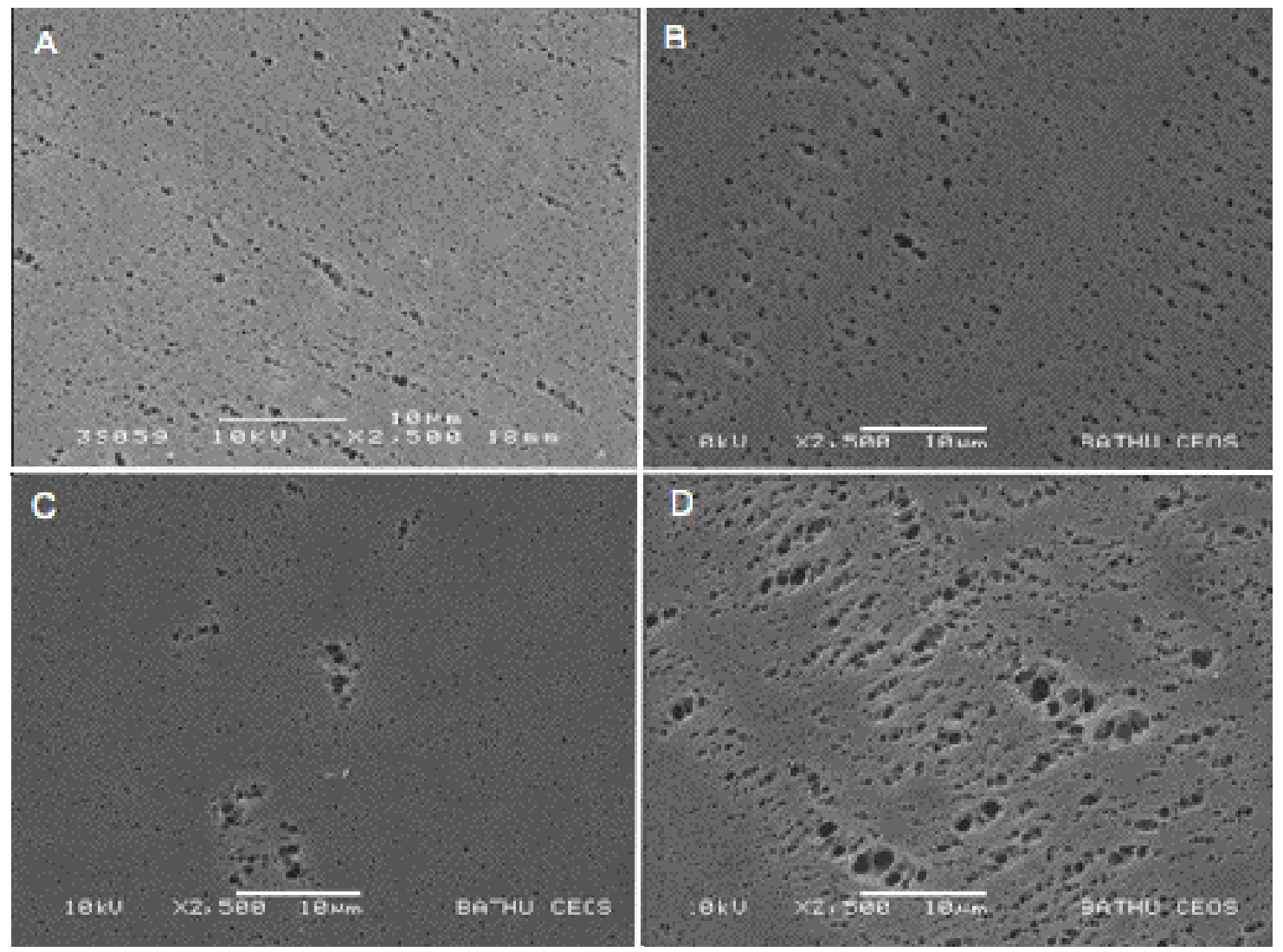


\subsection{Simvastatin Release Mechanisms Are Different for Dis-Sim and Sap-Sim}

The results showed a decrease in statin concentration in the membrane, from $0.9 \mu \mathrm{g} / \mathrm{mL}$ at $48 \mathrm{~h}$ to $0.5 \mu \mathrm{g} / \mathrm{mL}$ at $96 \mathrm{~h}$ (Figure 3). There was no significant difference between the release profiles for the $0.5 \mathrm{mg} / \mathrm{g}$ and $1.0 \mathrm{mg} / \mathrm{g}$ sap-sim membranes, and there was no significant difference in release rate over the 4 days, with values remaining between 0.3 and $0.4 \mu \mathrm{g} / \mathrm{mL}$.

Figure 3. Simvastatin net release-profiles. Samples of membrane were dried and the simvastatin extracted then quantified by HPLC, and relative concentrations calculated from a calibration curve (not shown); $\mathrm{n}=6$.

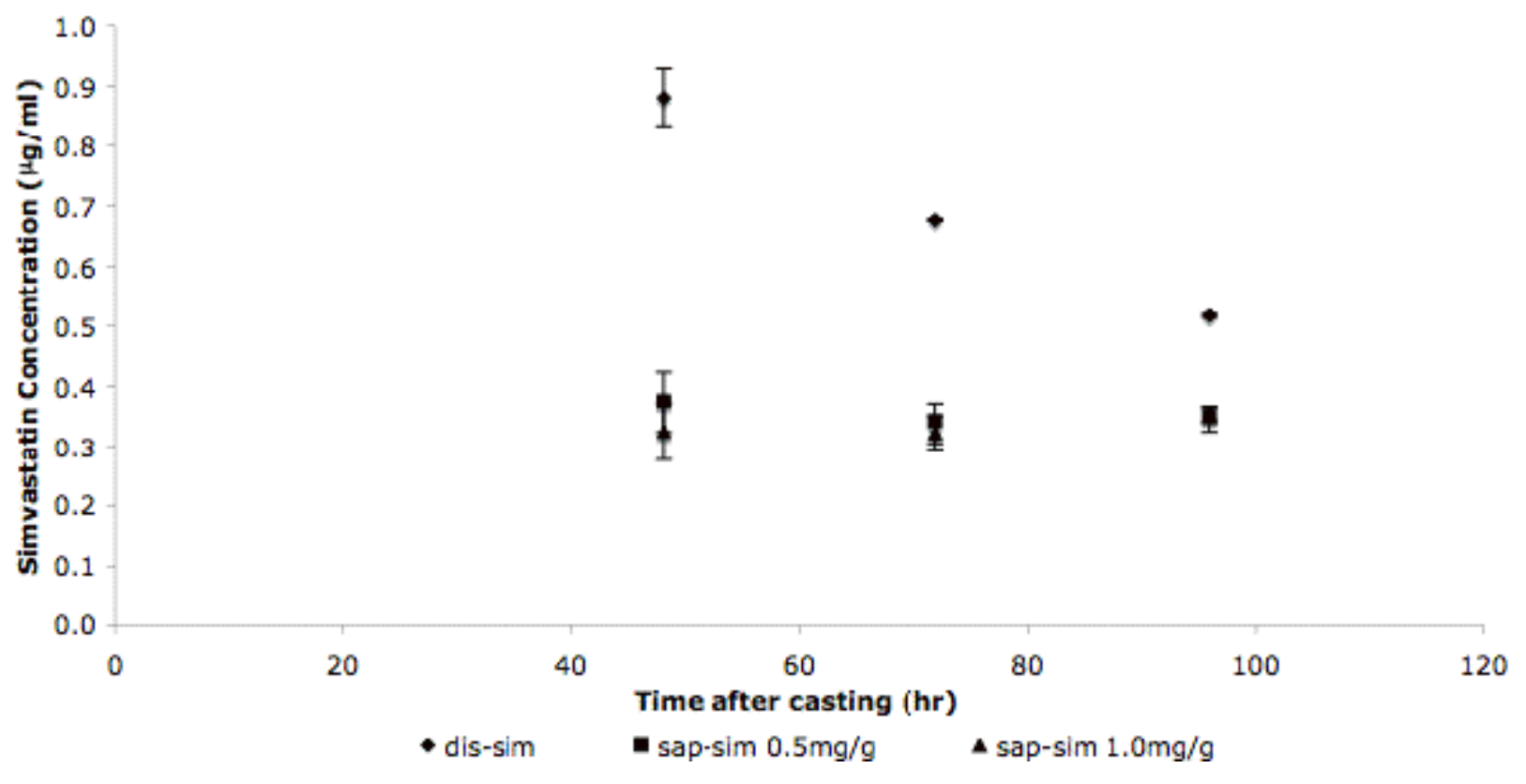

The decrease in viscosity of the casting dope and the increase in porosity and pore size that was observed on the addition of sap-sim can be attributed to either cleavage of PLGA ester bonds or a change in polymer chain structure due to the covalent bonding of the sap-sim, or a combination of both. The proposed mechanism for covalent bonding to the PLGA via cleavage of the ester bonds is shown in Figure 4.

The observation that there was no difference between the different concentrations of sap-sim is not surprising. The active statin is covalently bound to the PLGA membrane and thus there are two mechanisms to be considered in the release of the drug. There is the reaction required to release the statin, followed by diffusion of the statin to the bulk solution. It is likely that that there is a change in the controlling mechanism with release time, moving from reaction control initially, to diffusion control at later times. The diffusion of statin to the bulk solution will be dependent on the free concentration of statin in the bulk solution, and it is likely that this becomes limiting at relatively low concentrations. In contrast, the dis-sap membrane exhibited a typical diffusion-dependant pattern of drug release. 
Figure 4. Schematic of proposed mechanism of PLGA ester bond cleavage in presence of saponifiedsimvastatin and subsequent covalent bonding of PLGA and saponifiedsimvastatin.
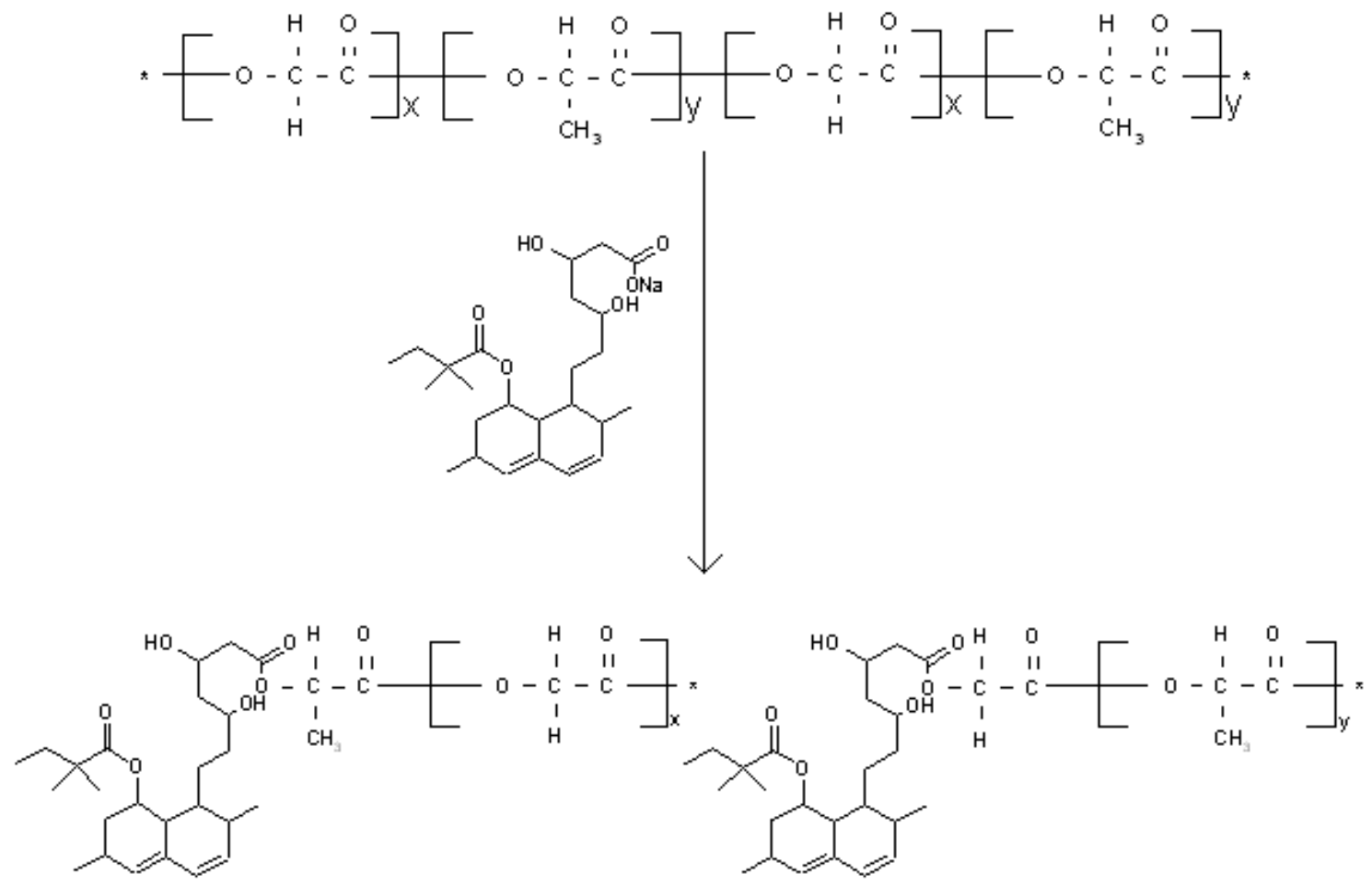

\section{Conclusions}

Simvastatin can be incorporated into PLGA membrane scaffolds by adding the drug into the casting dope. The release kinetics were shown to be dependent on the form of simvastatin and its interaction with PLGA on addition to the membrane casting dope. This study showed that simvastatincan be successfully incorporated into PLGA membrane scaffolds for controlled release of the drug into the bone microenvironment.

\section{Acknowledgements}

We would like to thank Fernando Acosta in the Department of Chemical Engineering and Ursula Potter in the Centre for Electron Optical Studies for their technical assistance, and Marie Curie for funding.

\section{References}

1. Jadhav, S.B.; Jain, G.K. Statins and osteoporosis: New role for old drugs. J. Pharm. Pharmacol. 2006, 58, 3-18.

2. Canalis, E.; Hott, M.; Deloffre, P.; Tsouderos, Y.; Marie, P.J. The divalent strontium salt S12911 enhances bone cell replication and bone formation in vitro. Bone 1996, 18, 517-523.

3. Buehler, J.; Chappuis, P.; Saffar, J.L.; Tsouderos, Y.; Vignery, A. Strontium ranelate inhibits bone resorption while maintaining bone formation in alveolar bone in monkeys (Macaca fascicularis). Bone 2001, 29, 176-179. 
4. Mundy, G.; Garrett, R.; Harris, S.; Chan, J.; Chen, D.; Rossini, G.; Boyce, B.; Zhao, M.; Gutierrez, G. Stimulation of bone formation in vitro and in rodents by statins. Science 1999, 286, 1946-1949.

5. Garrett, R.; Mundy, G. The role of statins as potential targets for bone formation. Arthritis Res. 2002, 4, 237-240.

6. Phillips, B.; Belmonte, N.; Vernochet, C.; Ailhaud, G.; Dani, C. Compactin enhances osteogenesis in murine embryonic stem cells. Biochem. Biophys. Res. Commun. 2001, 284, 478-484.

7. Garrett, I.R.; Esparza, J.; Chen, D.; Zhao, M.; Gutierrez, G.; Escobedo, A.; Horn, D.; Mundy, G.R. Statins mediate their effects on osteoblasts by inhibition of HMG-CoA reductase and ultimately BMP-2. J. Bone Miner. Res. 2000, 15, S225-S225.

8. Maeda, T.; Kawane, T.; Horiuchi, N. Statins augment vascular endothelial growth factor expression in osteoblastic cells vial inhibition of protein prenylation. Endocrinology 2003, 144, 681-692.

9. Sugiyama, M.; Kodama, T.; Konishi, K.; Abe, K.; Asami, S.; Oikawa, S. Compactin and simvastatin, but not pravastatin, induce bone morphogenetic protein-2 in human osteosarcoma cells. Biochem. Biophys. Res. Commun. 2000, 271, 688-692.

10. Garrett, I.R.; Escobedo, A.; Esparza, J.; Mundy, G.R. Cerivastatin increases BMP-2 expression in vivo and bone formation in concentrations of two orders of magnitude lower than other statins. J. Bone Miner. Res. 1999, 14, S180-S180.

11. Maeda, T.; Matsunuma, A.; Kawane, T.; Horiuchi, N. Simvastatin promotes osteoblast differentiation and mineralization in MC3T3-E1 cells. Biochem. Biophys. Res. Commun. 2001, 280, 874-877.

12. Woo, J.T.; Kasai, S.; Stern, P.H.; Nagai, K. Compactin suppresses bone resorption by inhibiting the fusion of prefusion osteoclasts and disrupting the actin ring in osteoclasts. J. Bone Miner. Res. 2000, 15, 650-662.

13. Von Stechow, D.; Fish, S.; Yahalom, D.; Bab, I.; Chorev, M.; Müller, R.; Alexander, J.M. Does simvastatin stimulate bone formation in vivo? BMC Musculoskel. Disord. 2003, doi: 10.1186/14712474-4-8.

14. Bauer, D.C. HMG CoA reductase inhibitors and the skeleton: A comprehensive review. Osteoporos. Int. 2003, 14, 273-282.

15. Gasser, J.A. Fluvastatin and cerivastatin are not anabolic for bone after local or systemic administration of non-toxic doses in mice and rats. J. Bone Miner. Res. 2001, 16, S295-S295.

16. Yao, W.; Li, C.Y.; Farmer, R.W.; Chen, J.L.; Mo, A.; Cooper, R.; Chmielewski, P.; Setterberg, R.B.; Lee, W.S.S.; Lundy, M.W. Simvastatin did not prevent bone loss in ovariectomized rats. J. Bone Miner. Res. 2001, 16, S294.

17. Maritz, F.J.; Conradie, M.M.; Hulley, P.A.; Gopal, R.; Hough, S. Effect of statins on bone mineral density and bone histomorphometry in rodents. Arterioscler. Thromb. Vasc. Biol. 2001, 21, 1636-1641. 
18. Mulder, M. Basic Principles of Membrane Technology; Kluwer Academic Publishers: Dordrecht, The Netherlands, 1992.

19. Whang, K.M.; Grageda, E.; Khan, A.; Mcdonald, J.; Lawton, M.; Satsangi, N. A novel osteotropic biomaterial OG-PLG: In vitro efficacy. J. Biomed. Mater. Res. A 2005, 74A, 247-253.

20. Crawford, D.T.; Qi, H.; Chidsey-Frink, K.L.; Simmons, H.A.; Thompson, D.D.; Ke, H.Z. Statin increases cortical bone in young male rats by single, local administration but fails to restore bone in ovariectomized (OVX) rats by daily systemic administration. J. Bone Miner. Res. 2001, 16, S295-S295.

21. Whang, K.; Zhao, M.; Qiao, M.; Rossini, G.; Horn, D.; Garrett, I.R.; Mundy, G.R.; Chen, D. Administration of lovastatin locally in low doses in a novel delivery system induces prolonged bone formation. J. Bone Miner. Res. 2000, 15, F380.

22. Ellis, M.J.; Chaudhuri, J.B. Poly(lactic-co-glycolic acid) hollow fibre membranes for use as a tissue engineering scaffold. Biotech. Bioeng. 2007, 96, 177-187.

23. Whang, K.M.; McDonald, J.; Khan, A.; Satsangi, N. A novel osteotropic biomaterial OG-PLG: Synthesis and in vitro release. J. Biomed. Mater. Res. A 2005, 74A, 237-246.

24. Piskin, E.; Isoglu, E.; Bölgen, N.; Vargel, I.; Griffiths, S.; Çavusoglu, T.; Korkusuz, P.; Güzel, E.; Cartmell, S. In vivo Performance of simvastatin loaded electrospun spiral-wounded polycaprolactone scaffolds in reconstruction of cranial bone defects in the rat model. J. Biomed. Mat. Res. A 2009, 15, 1137-1151.

25. Yang, S.F.; Leong, K.F.; Du, Z.H.; Chua, C.K. The design of scaffolds for use in tissue engineering. Part 1. Traditional factors. Tissue Eng. 2001, 7, 679-689.

26. Benoit, D.S.W.; Nuttelman, C.R.; Collins, S.D.; Anseth, K.S. Synthesis and characterization of a fluvastatin-releasing hydrogel delivery system to modulate hMSC differentiation and function for bone regeneration. Biomaterials 2006, 27, 6102-6110.

27. Shearer, H.; Ellis, M.J.; Perera, S.P.; Chaudhuri, J.B. Effects of common sterilization methods on the structure and properties of poly(D,L lactic-co-glycolic acid) scaffolds. Tissue Eng. 2006, 12, 2717-2727.

(C) 2010 by the authors; licensee MDPI, Basel, Switzerland. This article is an open access article distributed under the terms and conditions of the Creative Commons Attribution license (http://creativecommons.org/licenses/by/3.0/). 